\title{
Optical Properties in Biopolymers: Lignin Fragments
}

\author{
P. J. Salazar-Valencia, S. T. Pérez-Merchancano, and L. E. Bolívar-Marinéz \\ Departamento de Física, Universidad del Cauca, Calle 5 \# 4-70 Campus Tulcan, Popayán, Cauca, Colombia
}

Received on 8 December, 2005

\begin{abstract}
The lignin is an extremely challenging natural material with wide and interesting applications, during the last two decades, great efforts have been dedicated to the study of its optical properties. About this subject have occurred many controversial opinions because of the nature and interactions of this material with other components of the vegetal cellular wall. In this work we will explore and simulate the electronic absorption and vibrations spectrums based on the semiempirical geometrical and quantum mechanics optimizations of amorphous two and tree units softwoods lignin fragments of Coniferyl Alcohol. These fragments were previously obtained using the semiempirical methods, Modified Neglect of Diatomic Overlap MNDO and Zerner's spectroscopic version of the Intermediate Neglect of Differential Overlap in configuration interactions mode: ZINDO/S CI.
\end{abstract}

Keywords: Lignin; Biopolymers; Optical properties; Semiempirical methods

\section{INTRODUCTION}

The lignin is a highly complex biopolymer which is considered the second biggest source of carbon in the planet after the hydrocarbons. It undergoes vital functions for the terrestrial plants structural strength, conduction of water and defence against pathogen agents. Despite this roles, the study of lignin has been limited by its own nature, the fact that the macromolecular pattern can vary from species to species has make almost impossible to generate a detailed model of it. $[1,2]$

The chemical and technological aspects of lignin modification products are huge, beginning with the great amounts of raw material. All these products are available from the paper production industries that are usually disposed or burn as a secondary energy source; they have great energy content because of the presence of aromatic rings, and besides the carbon skeleton presents a wide range of substitution and addition reaction sites.

These products also have great compatibility with basic chemicals like Sulphur, Sodium and Nitrogen; they also have good absorbent, adhesion and ion exchange properties, as well they are a direct source of various kinds of phenolic and aromatic compounds. [3]

The main studies of lignin properties have been carried out on samples obtained from the processes of wood pulp extraction in the production of paper [4]. The conditions of the obtained material do not reflect the true nature of lignin due to the highly destructive procedures required to separate the cellulose fibbers that lignin binds together. [5] For these reason, studies of the lignin properties are easier to be develop from the study of its precursors or fragments obtained from the basic reaction outlines, which have already been identified.

In softwoods the most abundant precursor is the Coniferyl Alcohol (90-75\%) [6] and the preferred polymerization mechanisms are a free radical ones, called $\beta-O-4$ (50\%), $\beta-5$ (9$12 \%)$, and 5-5 (10-11\%)[7]. These structures are named according to the reactions sites that the formation of a specific structure involves, these patterns and reactions define and regulate the entire structure at the macromolecular level. [8]

We believe that these linkages, which join the precursor units in small chains (Fig. 1. and Fig. 2.), from two to six units, and these chains among themselves, contain all the necessary information to understand some basic characteristics of the lignin properties.

Through the use of semiempirical methods we will explore some of the energetic and spectroscopic characteristics of these structures.

\section{METHODOLOGY}

We can find in the literature the molecular structure of the precursors and the reaction patterns that lead to the most abundant polymeric products in lignin. These structures can be created with the free radical pathway which is the preferred polymerization mechanism in lignin biosynthesis, although only a few of them have significant presence in the macromolecular pattern [9].

We will explore the $\beta-O-4, \beta-5$, and 5-5 patterns as seen in Fig. 1 and Fig. 2, for two and three units by simulating the vibrational or infrared spectra and the electronic or ultraviolet spectra to gain insight into the possibilities and characteristics of theses molecules.

All the studied structures in this work have been previously obtained by a geometric optimization using the semiempirical method, Modified Neglect of Diatomic Overlap or MNDO. The simulation of the vibrational or infrared spectra will be obtained with a vibration calculation with the MNDO method.

On the other hand, the poor quality in the treatment of the excited states and electron correlation which is inherent to the MNDO method makes necessary to use the Zerner's spectroscopic version of the Intermediate Neglect of Differential Overlap in configuration interactions mode: ZINDO/S CI to the simulation of the electronic or ultraviolet spectra, with a 3 occupied and 3 unoccupied orbital criterion. All the calculation will be performed with the computational package HyperChem 5.11 of the Hypercube house [10].

All these methodologies have been extensible tested and are known to be pretty accurate and reliable in the study of organic molecules. [11] 


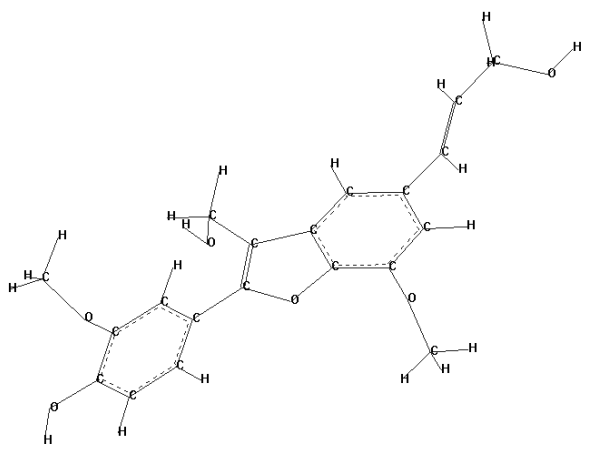

(a)

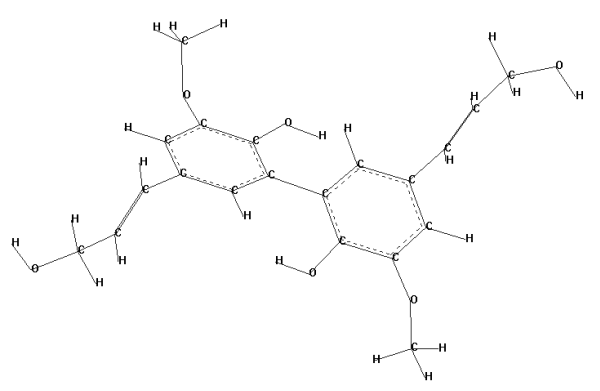

(b)

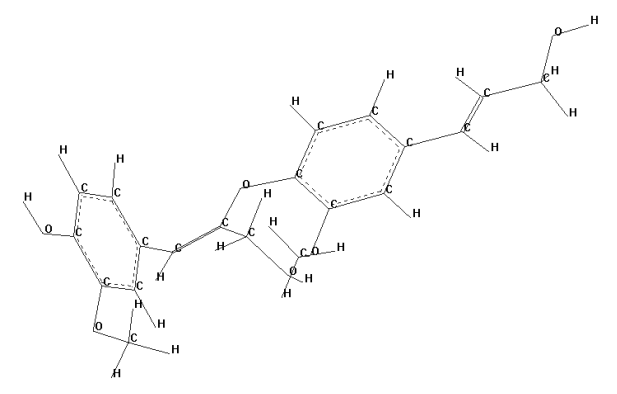

(c)

FIG. 1: Structures of 2 Coniferyl Alcohol units, (a) $\beta-5$, (b) 5-5, and (c) $\beta$-O-4 linkages.

\section{RESULTS AND ANALYSIS}

In the Table 1 we show the heat of formation values obtained from the geometry optimization for the studied structures. Although the 5-5 linkage offers the lower values, we can clearly see that there is no possibility for this linkage to be predominant in the structural patter of lignin. This type of union, a Carbon-Carbon one, will not allow a polymerization of more than two units because it closes the structure preventing that a third unit could be link to any of the carbons in the reaction sites.

The reason, because the carbon $\left(1 \mathrm{~s}^{2} 2 \mathrm{~s}^{2} 2 \mathrm{p}^{2}\right)$ can join to four more carbons and in a polymerization by the 5-5 mechanism a third unit will find completed the octet in the reaction site.

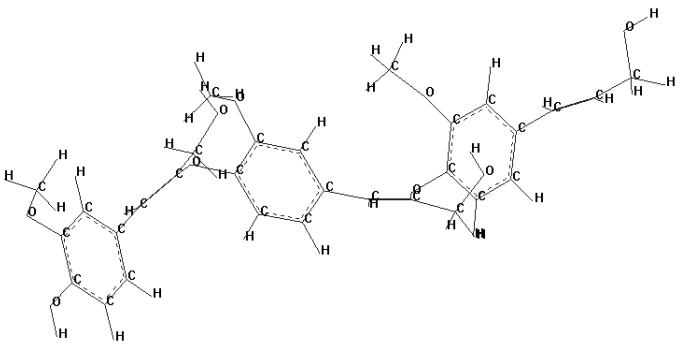

(a)

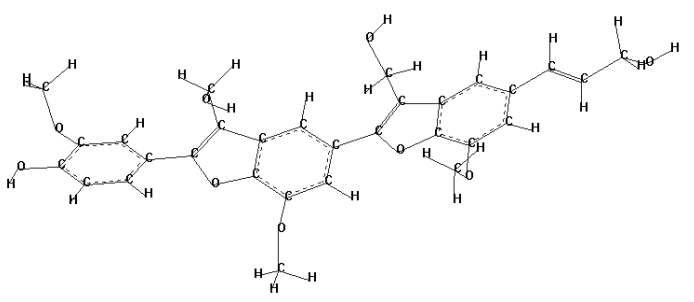

(b)

FIG. 2: Structures of 3 Coniferyl Alcohol units, (a) $\beta-O-4$, and (b) $\beta-5$ linkages.

TABLE I: Heat of formation for the structures studied, in $\mathrm{kcal} / \mathrm{mol}$.

\begin{tabular}{|l|l|l|}
\hline Linkage & 2 units & 3 units \\
\hline$\beta-\mathrm{O}-4$ & $-179,470$ & $-260,841$ \\
\hline$\beta-5$ & $-186,723$ & $-273,067$ \\
\hline $5-5$ & $-197,492$ & - \\
\hline
\end{tabular}

Even with this disadvantage this linkage undergoes a very important role in the structural pattern of lignin. This process has been identified as the mechanism that binds together the big polymeric chains.

The $\beta-5$ structure offers the next best value but this one does not appear very frequently in the structural pattern. This is an special type of linkage with a combines a Carbon-Carbon and a Carbon-Oxygen bridges, in theory this would allow the growth of bigger chains but all the studies have shown that this structure appears only linking small condensed units to the bigger chains [12].

The structure with the highest heat of formation value is the $\beta-\mathrm{O}-4$, which involves a Carbon-Oxygen union. This ones is the most frequently found in the structural pattern of lignin and is in charge of creating the long polymeric chains by joining the precursor units as it is reported in literature [13].

In this structure the Carbon-Oxygen bridge allows the addition of any amount of units in structures that need less energy as they get bigger. This information can confirm the theoretical data that shows the $\beta-O-4$ linkage as the most abundant and stable in softwoods lignin. [14]

In the Fig. 3 we show the simulation of the vibrational or infrared spectra for three Coniferyl Alcohol units with $\beta$-O4 linkage, frequency in $\mathrm{cm}^{-1}$ and intensity in $\mathrm{km} / \mathrm{mol}$. We only show the simulation of one infrared spectrum because all 
the studies structures have almost have the same vibrational behaviour with the most intense peaks in almost the same positions. [15]

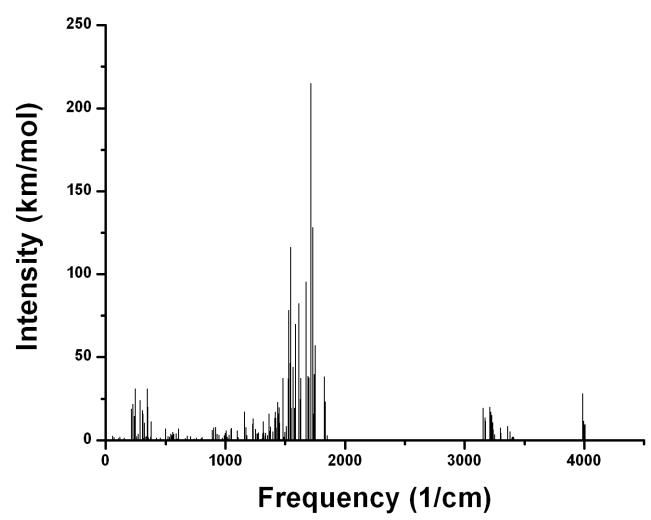

FIG. 3: Simulation of the infrared or vibrational spectra of tree Coniferyl Alcohol units with the $\beta$-O-4 linkage.

The most intense peaks are located in the $1750 \mathrm{~cm}^{-1}$ region related to the benzene rings vibrational activity; in the $1500 \mathrm{~cm}^{-1}$ region, the peaks are related to the vibrations of the linkages atoms: Carbon-Carbon (5-5), Carbon-Oxygen ( $\beta$ O-4) or mixed ( $\beta-5)$. The only difference is that the CarbonCarbon components are more active than the Carbon-Oxygen ones. Other intense peaks are located in the 250, 3200, and $4000 \mathrm{~cm}^{-1}$ regions corresponding to the vibrations of the $\mathrm{OH}$ and $\mathrm{OCH}_{3}$ groups, these ones are not involved in the formation of the linkages.

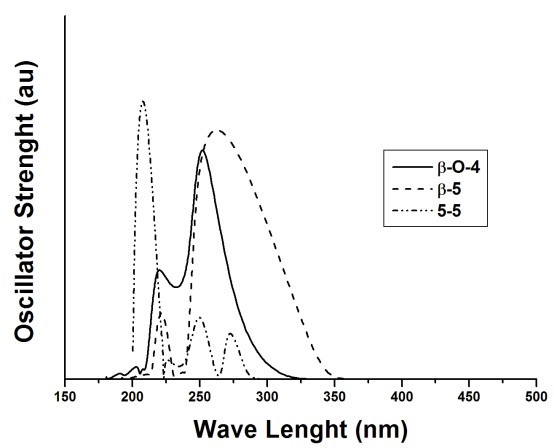

FIG. 4: Simulation of the electronic or ultraviolet spectra for the 2 Coniferyl Alcohol units, with $\beta-O-4, \beta-5$, and 5-5 linkages.

In the Fig. 4 we show the simulation of the electronic or ultraviolet spectra for the studied structures of 2 units in this work, wave length in nanometers and oscillator strength in arbitrary units. Most of the structures begin to absorb in the 180 $\mathrm{nm}$ region which correspond to a $7.2 \mathrm{eV}$ value that is in a good agreement with the experimental results. The most intense peaks are located in the $250 \mathrm{~nm}$ range $(5.2 \mathrm{eV})$ for the $\beta-O-4$ as reported, [16] $275 \mathrm{~nm}(4.7 \mathrm{eV})$ for the $\beta-5$, and $210 \mathrm{~nm}(6.2$
$\mathrm{eV})$ for the 5-5. All this transitions are in the range of one orbital below the highest occupied molecular orbital (HOMO) $|\mathrm{H}-1\rangle$ and one orbital above the lowest occupied molecular orbital (LUMO) $|\mathrm{L}+1\rangle$.

The peak separation in the 5-5 dimmer compared to the other two studied here can be explained in terms of its structural components. Here both benzene rings are very close to each other and the electronic transitions in the electronic clouds along with the highly resonant nature of the bonds leads the structure to begin absorbing energy earlier than the two others. This intense activity also creates a shielding effect on the carbon chains and other substituting groups.

Likewise, the $\beta-5$ dimmer has mores stability due to the unique combination of bonds whose behavior is very similar the one observed in the $\beta-\mathrm{O}-4$ structure where de separation of the benzene rings gives more possibilities to the rest of the molecule components to interact and react.

For the 3 units structures as we show in Fig. 5 the most intense peak for the $\beta-\mathrm{O}-4$ structure is located in the $260 \mathrm{~nm}$ $(5 \mathrm{eV})$ region, $15 \mathrm{~nm}$ less than in the previous case, getting nearer to the reported valued in literature. [17] We can infer that the peak will eventually converge to the $240 \mathrm{~nm}(5.4 \mathrm{eV})$ region where the analytical results locate it. The $\beta-5$ structure peak is in the $300 \mathrm{~nm}(4.3 \mathrm{eV})$ region but we must remember that there is very little chance of this structure to appear in the structural pattern. [18]

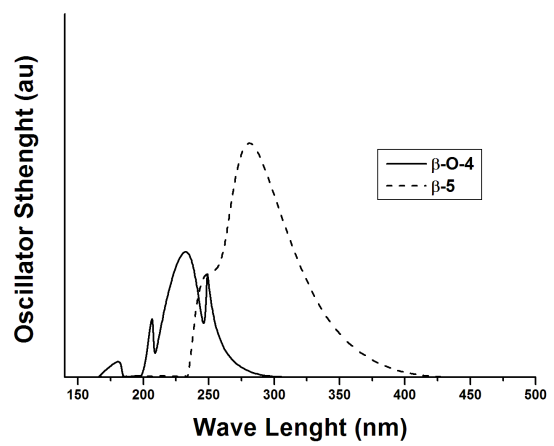

FIG. 5: Simulation of the electronic or ultraviolet spectra for the 3 Coniferyl Alcohol units, with $\beta-O-4$ and $\beta-5$ linkages.

The 5-5 structure is not possible for 3 units taking into account the reasons exposed earlier in this paper. Again, the peaks are associated with transitions in the range of one orbital below the highest occupied molecular orbital $|\mathrm{H}-1\rangle$ and one orbital above the lowest occupied molecular orbital $|\mathrm{L}+1\rangle$ this are $\sigma$ type orbitals due to its twisted shape.

The data obtained from the spectra simulations with the ZINDO/S method, as can be seen in the Figures 4 and 5, gives a pretty good idea about the band GAP of these structures. Our simulations predict an approximated value of 7.2 electron volts making these structures natural isolators. This data can be related to the role of lignin as an important part of the isolator nature of wood.

The band GAP can also be estimated with the energy difference between the HOMO and LUMO orbital energies, values 
that are corresponding to those estimated with the electronic spectra in this work.

\section{CONCLUSION}

In this work we have obtained new results about the electronic and optic behavior of softwood lignin, we expect this results to aid a deeper understanding of lignin properties and characteristics.

We have carried out the simulation of the optical characterization of softwoods lignin fragments of two and three units of Coniferyl Alcohol using the $\beta-\mathrm{O}-4, \beta-5,5-5$, linking mechanisms with the semiempirical methods MNDO and ZINDO/S CI.

Our results show that the preferred polymerization mechanism is the $\beta-O-4$ type due to the nature of the Carbon-Oxygen bonds; this geometry allows a long range growth of the poly- meric chains by lowering the heat of formation values.

The vibrational dominium is dominated by the structures aromatic part activity; the most intense peaks are located in the $1750 \mathrm{~cm}^{-1}$, a distinctive mark of the lignin according to the analytical reports.

The simulation of the infrared spectra, have shown also the predominance of the electronic activity of the aromatic components; the values of the band GAP found in these simulations are on $7 \mathrm{eV}$ order, value where the electronic spectra of the structures begins to absorb, this behavior is presumed to remain present as long as the structure of lignin grows.

\section{Acknowledgments}

The authors wish to thank the University of Cauca Vicerectory of Investigations "VRI" for the economic aid in the presentation of this work.
[1] M. Hofrinchter y A. Steinbüchel. Biopolymers, Vol. 1, Lignin, Humic Substances and Coal. Wiley - Vch. 2001.

[2] Carl J. Houtman, What Factors Control Dimerization of Coniferyl Alcohol? Holzforschung. Vol. 53, No. 6, (1999).

[3] Mikiji Shigematsu, et al. Journal of Computer Chemistry, Japan, Vol 1, No 4 (2002).

[4] Glasser, Wolfgang and Sarkanen, Simo. Lignin, Properties and Materials. ACS Symposium Series 397. American Chemical Society. Washington DC. Maple Press. 1989.

[5] C. J. Houtman, and R. H. Atalla, Plant Physiol. 107, 977 (1995)

[6] C. J. Houtman, Holzforschung, 53, 585-589

[7] M.J.S. Dewar, J. Am. Chem. Soc. 99, 4899 (1977).

[8] James J. P. Stewart, Optimization of parameters for semiempirical methods I. Method. Journal of Computational Chemistry, 10, 209 (1989).

[9] Walter M. Fabian, AM1 Calculations of rotation around essential single bonds and prefer conformations in conjugated molecules. Journal of Computational Chemistry, 9, 369 (1988).

[10] A. Jon Pople, et al. Approximate Molecular Orbital Theory. McGraw Hill Book Company, 1970

[11] W. P. Anderson, T R. Cundari, and M. C. Zerner, Int. J. Quan- tum Chem. 39, 31 (1991).

[12] J. C. Pew, Evidence of biphenyl group in lignin. J. Org. Chem. 28, 1048 (1963).

[13] N. L. Allinger, J. Am. Chem. Soc. 99, 8127 (1977).

[14] Soares Landim, Alan and Ruggiero, Reinaldo. Caracterização e Estudo da Fotodegradação de Poliguaiacois. Sintetizados por Catálise Oxidativa, Como Modelos. Macromoleculares de Lignina. Revista Horizonte Científico. Universidade Federal de Uberlândia (PIBIC's/UFU). 2002.

[15] Oxygen-alkali degradation of loblolly pine dioxane lignin: changes in chemical structure as a function of time of oxidation. Thomas E. Crozier. B.S. 1968, The Institute of Paper Chemnsitry University of California, Santa Barbara.

[16] A. Antonio Pizzi, Advanced Wood Adhesive Technologies. Chapter 6. ISBN 0-8247-9266-1. Marcel Dekker Editor.

[17] Li, Lixiong. Supercritical Fluid Extraction from lignin. Lignin Properties and Materials. ACS Symposium Series 397. Chapter 4. American Chemical Society. Washington 1989.

[18] D. Fengerl, G. Wegener, Wood Chemistry, Ultrastructure, Reactions. Walter de Gruyter: New York, 1947. 